\title{
Superior Training Efficacy of Beginning Movement Load Training for the Baseball Throwers
}

\author{
Wen-Yi Chou ( $\nabla$ murraychou@yahoo.com.tw) \\ Kaohsiung Chang Gung Memorial Hospital, Chang Gung University College of Medicine \\ Jih-Yang Ko \\ Kaohsiung Chang Gung Memorial Hospital, Chang Gung University College of Medicine \\ Shu-Fang Chen \\ Kaohsiung Chang Gung Memorial Hospital \\ Chia-Feng Wu \\ Kaohsiung Chang Gung Memorial Hospital \\ Kuan-Ting Wu \\ Kaohsiung Chang Gung Memorial Hospital \\ Shun-Wun Jhan \\ Kaohsiung Chang Gung Memorial Hospital
}

\section{Research Article}

Keywords: beginning movement load training, baseball, shoulder, thrower`s ten

Posted Date: May 27th, 2021

DOI: https://doi.org/10.21203/rs.3.rs-558327/v1

License: (c) (i) This work is licensed under a Creative Commons Attribution 4.0 International License.

Read Full License 


\section{Abstract}

Background : Superior shoulder motion with rotator cuff activation are essential for the performance of the throwing athletes. The present study compared the novel beginning movement load training (BMLT) and popular throwers ten program regarding the training efficacy of baseball throwers. We hypothesized that the BMLT contributed the superior training efficacy than popular throwers ten program.

Methods : Forty adult baseball players were randomized into study group and control group equally. In study group, the cyclic shoulder motion was repeatedly operated 3 days in a week and lasted for 6 weeks using three different BMLT training machines. As for control group, three popular cyclic training in the throwers ten program were adopted for the shoulder trainings as the same protocol in study group. The evaluations before and after training included the static range of motion (ROM), the maximal voluntary isometric contraction (MVICs) of the target muscle (pectoralis major, middle deltoid and supraspinatus) and throwing velocity.

Result : After 6-week course, study group had significant wider static ROM in saggital adduction $(p=0.034)$, coronal internal rotation $(p=0.038)$ and external rotation $(p=0.04)$ than in control group. The maximal voluntary isometric contraction (MVIC) ratio of middle deltoid/supraspinatus was significant lower in study group (Study: Control $=1.14 \pm 0.76: 3.56 \pm 5.57, p=0.044$ ) which indicated the enhanced supraspinatus maximal contraction in the study group after training. In addition, the study group had significant improvement in throwing speed $(117 \pm 10$ vs $109 \pm 10 \mathrm{Km} / \mathrm{hr}, \mathrm{p}=0.031)$.

Conclusion : The BMLT contributed the superiority in range of motion, recruitment of supraspinatus and throwing velocity than the popular thrower's ten program. It could be a favourable training for the overhead activity.

\section{Introduction}

Overhead motion is a very common activity in daily life. In addition, many sports involve overhead motion, such as baseball, javelin, tennis, badminton. Throwing motion involves giving velocity and direction to a specific target, and is accomplished by coordination of the legs, trunk, and arms, wellknown as the concept of a kinetic chain[1]. The throwing motion is generally divided into 5 phase[2] and many studies have revealed the important role of the cocking phase in the whole throwing motion, as it is related to the shoulder injury and performance[3-6]. It is believed that most throwing injuries occur in the cocking phase which contains the maximal external rotation of the shoulder and the subacromial impingement related supraspinatus tendon partial-thickness tears or even full-thickness tears might happen in this stage that has been reported to be the common sources of pain that limit the ability to throw $[7,8]$. Regardless of treatment after injury, many exercise modalities have been proposed to prevent injury or enhance the performance of the rotator cuff, especially the supraspinatus, including shoulder external rotation training using an elastic band or light-weight dumbbell, and Thrower's ten program[2, 9]. Concerning training that mimics the specific cocking phase without the potential complications of open 
kinetic chain training, a novel training concept with special equipment, beginning movement load training, is being introduced here.

Beginning movement load training (BMLT) and related training equipment were developed by $\mathrm{Dr}$ Koyamain 1994. In contrast to ordinary open kinetic chain training, in which muscle contraction occurs at the start of an exercise, the mainstay of BMLT is relaxing the muscle at the beginningof an exercise, followed by lengthening and contraction. This forms a cycle of motion in which an agonist muscle repeats relaxation-lengthening-shortening and in which co-contraction is prevented[10]. Another important characteristic of BMLT is rotation on a long axis at extension and flexion of the extremities, which is defined as the "dodge movement"[11]. With the dodge movement, a short-term relaxation interval emerges before a shortening contraction of an agonist muscle, preventing co-contraction[11]. BMLT employs a specific device composed of a cam-crank mechanism, which permits synchronization of openclosed chain motions in the horizontal direction, in addition to up-down motions in the vertical line, thereby increasing the joint range of motion combined with torsional motion. Using these machines, upper extremity training involves reciprocating motions in the vertical direction and rotation on the horizontal plane; moreover, horizontal extension, flexion, outward/inward rotation, external/internal rotation of the shoulder, extension, and flexion of the elbow, and pronation/supination of the forearm are executed simultaneously (Fig. 1-3). Compared with conventional open chain training[12], this specific training modality simulates common overhead activities, such as the cocking phase of the overhead throwing, which combine the characteristics of open and closed kinetic chains. According to the training rationale and activity, we assert that BMLT is a favorable training modality for the shoulder in light of its complicated muscle activation. This study aimed to compare the differences and outcomes of BMLT and the conventional Thrower's ten program training for the cocking phase motion (abduction with maximal external rotation) of the baseball throwers. We hypothesized that the BMLT contributed the superior training outcomes, including range of motion, cuff recruitment and velocity, than popular throwers ten program.

\section{Materials And Methods 2.1 Participants}

This prospective comparative randomized study was approved by the Institutional Review Board (IRB) of Chang Gung Medical Foundation (IRB Study No. 201600644B0). Forty adult, asymptomatic and active amateur baseball players were enrolled in this study. These athletes were randomized in equal numbers into the study group and control group. Players with musculoskeletal disorders in the last 3 months were excluded due to safety concerns. All the evaluators and training were performed in sports performance training center of our hospital. All participants read and signed an informed consent form approved by the Institutional Review Board of our hospital.

\subsection{Evaluations}


Pre- and post-training evaluation of the throwing shoulders included measurement of static range of motion (ROM), dynamic surface electromyography of pectoralis major, middle deltoid and supraspinatus, and throwing velocity. All these parameters were recorded before and after 6-week training.

\subsubsection{Static range of motion}

The shoulder range of motion related to throwing activity was recorded before and six weeks after the training. The goniometer was adopted to record shoulder ROM included saggital flexion/extension, coronal abduction/adduction, and external/internal 90-degree coronal abduction. All the measurements were conducted by one sports-specialized physical therapist that had involved in the study for more than three years.

\subsubsection{Throwingvelocity}

Following adequate and personal habitual dynamic stretch warm-up (reaching at least $60 \%$ of the maximum heart rate), the players were asked to throw five pitches with their greatest effort from the flat ground to a target net 18 meters away that simulate the distance from the pitcher plate to the catcher, and the highest pitch velocity was recorded. Throwing velocity was measured using speed radar gun (Stalker sport 2 radar gun, Stalker, USA).

\subsubsection{Dynamic surface electromyography}

Surface electromyography (sEMG) was adopted to assess muscle recruitment and outcomes under different training modalities. The maximal voluntary isometric contractions (MVICs, Newton meter, Nm) of the affected muscles were investigated by performing sequential motion mimicking the cocking phase on the different training machines. The muscles that were investigated that contribute to the cocking phase were the pectoralis major, middle deltoid and supraspinatus. To minimize cross-interference in the myoelectrical signal, the investigation was limited to these 3 muscles simultaneously. Muscle activity was measured using surface electromyography (DTS Belt Receiver/Retransmitter, DTS EMG Probe, Software: MR3, Noraxon, USA). Skin preparation of the affected shoulder was performed prior to sEMG sensor tagging. The pectoralis major was located medial to the axillary fold while the athlete medially rotated the arm against resistance. The electrodes were placed horizontally on the chest wall over the muscle approximately $6 \mathrm{~cm}$ below the clavicle. The electrodes for the middle deltoid were placed on the lateral aspect of the upper arm and $3 \mathrm{~cm}$ below the acromion, parallel to the muscle fibers. The myoelectrode signal of the supraspinatus was detected by placing of the electrode just above the spine of the scapula and $2 \mathrm{~cm}$ from its medial border. Players in the study group were asked to perform shoulder 90-degree coronal abduction with external rotation from 0 degrees to maximum horizontal adduction on the BMLT machine (SCA. BACKS 4D-2000, Worldwing, Tottori, Japan) under a $5 \mathrm{Kg}$ weight load. The motion simulated the shoulder motion in the cocking phase. Players in the control group were also asked to perform the same motion, but on a commercialized Cable machine (Dual adjustable pully L370, BH, Spain).

\subsection{Training in the study group}


In the study group, players were asked to discontinue all upper extremity training except BMLT using the Scapula 2000, Scapula 1000 and Clavicle 2000 machines (Fig. 1-3). It also emphasized concomitant scapular motion from the upward rotation to scapular retraction. The training sequence of Scapula 2000 started from the position of Fig. 1 a to $1 \mathrm{c}$ on a single shoulder. The Scapula 1000 (Fig. 3b) employed the same sequence as the Scapula 2000, with the exception that both upper extremities operated simultaneously. The training on Clavicle 2000 stared from the position of Fig. 2a followed by Fig. 2b. All the sequences emphasized the relaxation in the starting position followed by lengthening and contraction of shoulder. On each machine, the cyclic motion was repeated 15 times per session; the player completed 5 sessions on each machine per day, 3 days a week. BMLT lasted for 6 weeks: it started with a $0 \mathrm{Kg}$ load on each machine in the first week, which was increased to $5 \mathrm{Kg}$ in the $2 \mathrm{nd}$ week, followed by increases of $5 \mathrm{Kg}$ per week until reaching $25 \mathrm{Kg}$ in the 6th week. The total weight-load during training was $10,125 \mathrm{Kg}$ over the 6-week course.

\subsection{Training in the control group}

Conventional training was carried out using 3 popular cyclic motions, as adopted in the Thrower's ten program[12] using a Cable machine, which included shoulder diagonal pattern flexion/extension, shoulder abduction/scaption, and external/internal rotation at a 90-degree coronal abduction. Each cyclic motion was repeated 15 times in one session, with 5 sessions for each cyclic motion being completed in one day, 3 days a week. All the motions operated in full motion of the affected shoulder and the training finished within 90 minutes in a day. Conventional training also lasted for 6 weeks: it started with a $0 \mathrm{Kg}$ load for each motion in the first week, then the weight was increased to $5 \mathrm{Kg}$ in the 2nd week, followed by increases of $5 \mathrm{Kg}$ per week, reaching $25 \mathrm{Kg}$ in the 6 th week. The total weight-load over the 6-week training period was $10,125 \mathrm{Kg}$ that was the same as in the study group.

\subsection{Statistical analysis}

Statistical analysis was performed using SPSS for Windows (Statistical Package for the Social Sciences, version 22.0; SSPS Inc., Pompano Beach, FL, USA). The Wilcoxon test was adopted to analyze the pretraining and post-training conditions, and comparison of the study group with the control group was performed using the Mann-Whitney $U$ test. A value of $p<0.05$ was accepted as indicative of statistical significance.

\section{Results}

Forty active college baseball players were recruited to participate in this prospective randomized comparative study. Twenty players were assigned to the study group, and twenty to the control group. No significant differences in the pre-training demographic characteristics were observed between groups (Table 1). 
Table 1

Athlete demographic characteristics.

\begin{tabular}{|lllll|}
\hline & Study group & Control group & Total & P-value \\
\hline Number of athletes & 20 & 20 & 40 & 1.0 \\
\hline Dominant hand (R/L) & $16 / 4$ & $18 / 2$ & $34 / 6$ & 0.661 \\
\hline Ave. age (years) & & & & \\
\hline Mean \pm SD & $22.2 \pm 3.3$ & $21.1 \pm 1.7$ & $21.6 \pm 2.6$ & 0.179 \\
\hline (Range) & $(20-33)$ & $(20-26)$ & $(20-33)$ & \\
\hline Gender (male/female) & $20 / 0$ & $20 / 0$ & 40 & 1.0 \\
\hline 1B & 1 & 3 & 4 & \\
\hline 2B & 2 & 1 & 3 & \\
\hline 3B & 2 & 0 & 2 & \\
\hline IF & 3 & 4 & 7 & 0.851 \\
\hline EF & 3 & 3 & 6 & \\
\hline Pitcher & 8 & 8 & 16 & \\
\hline Catcher & 1 & 1 & 2 & \\
\hline
\end{tabular}

\subsection{Static range of motion}

No difference in the static ROM of the shoulder before training was observed between groups. After 6 weeks training, the study group revealed significant increases in saggital extension $(p=0.009)$, coronal adduction $(p=0.004)$ and 90-degree coronal abduction with external $(p<0.001) /$ internal rotation $(p=$ $0.001)$ as compared with the pre-training static ROM, while significant improvements were observed incoronal adduction $(p=0.045)$ and 90-degree coronal abduction with internal rotation $(p=0.045$, Table 2 ) in the control group. The study group demonstrated a significantly greater ROM in saggital extension $(p=0.034)$ and coronal internal rotation $(p=0.038) /$ external rotation $(p=0.04)$ than the control group. The results of the present study showed that BMLT resulted in a greater static ROM than conventional training in shoulder saggital extension and external/internal rotation. 
Table 2

Comparative outcomes of shoulder static range of motion and throwing speed.

Study group

(1) Flexion(o)

Pre-training

Post-training

P-value $e^{2}$

(2) Extension(o)

Pre-training

Post-training

P-value ${ }^{2}$

(3) Abduction(o)

Pre-training

Post-training

P-value ${ }^{2}$

(4) Adduction(०)

Pre-training

Post-training

P-value ${ }^{2}$

(5) Internal rotation

Pre-training

Post-training

P-value ${ }^{2}$

(6) External rotation

Pre-training
Control group

P-value $^{1}$

0.989

1.0

0.180

0.180

$179 \pm 2(170-180)$

$180 \pm 0(180)$

$180 \pm 0(180)$

$179 \pm 5(160-180)$

$61 \pm 14(35-100)$

0.310

$64 \pm 10(50-95)$

$62 \pm 10(40-70)$

0.034

$70 \pm 9(55-90)$

0.320

0.009

0.320

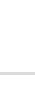




\begin{tabular}{|llll|}
\hline & Study group & Control group & P-value $^{1}$ \\
\hline Post-training & $120 \pm 16(85-145)$ & $111 \pm 12(75-125)$ & 0.040 \\
\hline P-value ${ }^{2}$ & $<0.001$ & 0.194 & \\
\hline$(7)$ Speed (KPH) & $114 \pm 9(98-128)$ & $108 \pm 9(90-125)$ & 0.088 \\
\hline Pre-training & $117 \pm 10(100-132)$ & $109 \pm 10(94-130)$ & 0.031 \\
\hline Post-training & 0.001 & 0.411 & \\
\hline P-value & & & \\
\hline $\begin{array}{l}\text { P-value } \\
\text { test. }\end{array}$ & & & \\
$\begin{array}{l}\text { P-value } \\
\text { Wilcoxon test. }\end{array}$ & & \\
\hline
\end{tabular}

\subsection{Dynamic surface electromyography}

The maximal voluntary isometric contractions (MVICs) of the pectoralis major, middle deltoid and supraspinatus were recorded for analysis of the differences in the muscle recruitment pattern and outcomes following the different training modalities.

The MVIC in pectoralis major were no significantly differences in both groups before and after the training (Fig. 4a). The MVIC of middle deltoid and supraspinatus were significantly higher in the control group before training and became statistically insignificance after training (Fig. 4b\&c). In addition, the ratio of the middle deltoid versus the supraspinatus became significantly higher in control group after six weeks training (control group vs. study group $=3.56 \pm 5.57$ vs. $1.14 \pm 0.76, p=0.044$, Fig. $4 d$ ) which indicated that BMLT offered the superior supraspinatus training efficacy than conventional training regarding the synchronized motion of middle deltoid and supraspinatus in cocking motion.

\subsection{Throwing velocity}

In the analysis of throwing performance, throwing speed is one of the parameters that may reflect the outcome after training. In the present study, the pre-training throwing speed was not significantly different between groups $(p=0.088)$; however, differences were observed after 6 weeks of training. The study group exhibited significant improvement in pitching velocity as compared with the control group (117 \pm 10 vs. $109 \pm 10 \mathrm{Km} / \mathrm{hr}, \mathrm{p}=0.031$ ).

\section{Discussion}

To the best of our knowledge, this was the first comparative study to analyze the training outcomes of BMLT for the throwers' shoulder than popular thrower's ten program. The principal findings of the present 
study were that this BMLT yielded a greater range of motion, supraspinatus recruitment in the shoulder cocking motion, and improved the throwing velocity for the baseball players. Hence, the results implied that BMLT is an applicable training alternative for the baseball throwers or overhead activities.

The results of the present study showed that BMLT produced a greater static ROM than conventional training in shoulder saggital extension and external/internal rotation, which is an effect of the most critical feature of BMLT, the three-dimensional training plane. This involves greater scapular movement in the cyclic motion from shoulder elevation to horizontal abduction. In the Thrower's ten program, most of the resistance or muscular training is carried out on a single plane of motion. Combining the reciprocal "dodge movement" on a stable-handled platform, the characteristics of open and closed kinetic chain exercise are merged, and BMLT increases safety and results in less training-related delayed-onset muscle soreness. Previous studies have demonstrated enhancement of training actions and functional activities by BMLT exercise with a greater range of motion $[10,13]$. The results of the present study also revealed that a greater range of motion was obtained using this novel weight training modality.

Synchronization of the middle deltoid and supraspinatus is believed to be critical during shoulder abduction $[14,15]$ especially in cocking phase. In general, the deltoid muscle plays a major role in shoulder abduction, rather than the supraspinatus. However, the supraspinatus lesion is a much more common injury than the deltoid and the conventional training is difficult in changing rotator cuff activation[16]. BMLT offered the superior supraspinatus MVICs (Fig. 4) than conventional training in cocking motion 6 weeks after training. The superior supraspinatus contractions were believed to be positive in improving rotation range of motion and performance in the overhead activity.

The middle deltoid contributes most to movement, while the shoulder abduct in internal rotation and abducts horizontally with external rotation[17-19]. The co-operative mechanism contributes most greatly in the cocking phase[17-19]. The supraspinatus works synchronously with the middle deltoid for shoulder abduction[20]. It has been reported that the supraspinatus is the common injured tendon in the throwing athletes, in particular, partial-thickness tears[7, 8]. Most of the strategies employed for the prevention of supraspinatus injury aim to enhance training or conditioning[21], introduce a proper throwing mechanism[22, 23], and specify an adequate throwing interval program with appropriate rest. As compared with conventional training, the contribution of the supraspinatus increased after 6 weeks of training which indicated that BMLT improved the supraspinatus contribution in the cocking motion over that resulting from conventional training. From the aspects of injury prevention and performance promotion, we postulated that BMLT is an alternative training to provide better supraspinatus training effect for the throwers. Meanwhile, the improvement of throwing velocity were observed in this study although the velocity improvement is multifactorial[24, 25], but it cannot be denied that the flexibility of the joint and rotator cuff strength posed the essential roles.

Limitations of the present study exist. First, the infraspinatus muscle, which is involved in shoulder external rotation, was not analyzed in the present study owing to the avoidance of EMG signal interference. Second, EMG analysis should also address muscles that facilitate scapular motion, such as 
the trapezius, rhomboids, levator scapulae and serratus anterior. Third, a longer training interval and recruitment of a greater number of athletes are required to demonstrate and corroborate the training outcomes.

\section{Conclusions}

The BMLT contributed the superiority in range of motion, recruitment of supraspinatus and throwing velocity than the popular thrower's ten program. We would like to conclude that BMLT is favourable alternative training modality for the baseball throwers.

\section{Abbreviations}

BMLT: Beginning movement load training; MVICs: maximal voluntary isometric contractions; ROM: Range of motion; sEMG: surface electromyography

\section{Declarations}

\section{- Ethics approval and consent to participate}

This prospective comparative randomized study was approved by the Institutional Review Board (IRB) of Chang Gung Medical Foundation (IRB Study No. 201600644B0). The patients were informed about the details of the study and provided written informed consent before study enrolment. Informed consent was obtainedfrom all the participants, and procedures were conducted according to the Declaration of Helsinki.

\section{- Consent for publication}

Written informed consent was obtained from the participants for publication of their individual details and accompanying images in this manuscript. The consent form is held by the authors and is available for review by the Editor-in-Chief.

\section{- Availability of data and materials}

All the data that support the results can be found in the manuscript.

\section{- Competing interests}

The authors have declared that no competing interests exist.

\section{- Funding}

There are no funds supporting this research. 


\section{- Authors' contributions}

WY contributed to planning of study, recruitment of the patients, Data interpretation, writing the draft, revised, analyzed data and statistics; JY and SF contributed to the design and literature review of the study, drafting revision; CF contributed to collecting data; KT and SW analyzed data and statistics. All authors read and approved the final manuscript.

\section{- Acknowledgements}

Grant was received in total or partial in the support of this study. The funding source is from Chang Gung Research Fund (No. CMRPG8F0881).

\section{References}

1. Chu SK, Jayabalan P, Kibler WB, Press J: The Kinetic Chain Revisited: New Concepts on Throwing Mechanics and Injury. PM R 2016, 8(3 Suppl):S69-77.

2. Weber AE, Kontaxis A, O'Brien SJ, Bedi A: The biomechanics of throwing: simplified and cogent. Sports Med Arthrosc Rev 2014, 22(2):72-79.

3. Douoguih WA, Dolce DL, Lincoln AE: Early Cocking Phase Mechanics and Upper Extremity Surgery Risk in Starting Professional Baseball Pitchers. Orthop J Sports Med 2015, 3(4):2325967115581594.

4. Roach NT, Venkadesan M, Rainbow MJ, Lieberman DE: Elastic energy storage in the shoulder and the evolution of high-speed throwing in Homo. Nature 2013, 498(7455):483-486.

5. Saka M, Yamauchi H, Yoshioka T, Hamada H, Gamada K: Scapular Kinematics During Late Cocking of a Simulated Throwing Activity in Baseball Players With Shoulder Injury: A Cross-Sectional Study Using a 3D-to-2D Registration Technique. J Sport Rehabil 2015, 24(2):91-98.

6. Takagi Y, Oi T, Tanaka H, Inui H, Fujioka H, Tanaka J, Yoshiya S, Nobuhara K: Increased horizontal shoulder abduction is associated with an increase in shoulder joint load in baseball pitching. $J$ Shoulder Elbow Surg 2014, 23(12):1757-1762.

7. Connor PM, Banks DM, Tyson AB, Coumas JS, D'Alessandro DF: Magnetic resonance imaging of the asymptomatic shoulder of overhead athletes: a 5-year follow-up study. Am J Sports Med 2003, 31(5):724-727.

8. Conway JE: Arthroscopic repair of partial-thickness rotator cuff tears and SLAP lesions in professional baseball players. Orthop Clin North Am 2001, 32(3):443-456.

9. Wilk KE, Meister K, Andrews JR: Current concepts in the rehabilitation of the overhead throwing athlete. Am J Sports Med 2002, 30(1):136-151.

10. Koyama Y, Kobayashi H, Suzuki S, Enoka RM: Enhancing the weight training experience: a comparison of limb kinematics and EMG activity on three machines. Eur J App/ Physiol 2010, 109(5):789-801. 
11. Koyama Y: Shin torēningu kakumei =: New training revolution, (Japanese Edition edn: Kodansha; 1991.

12. Wilk KE, Yenchak AJ, Arrigo CA, Andrews JR: The Advanced Throwers Ten Exercise Program: a new exercise series for enhanced dynamic shoulder control in the overhead throwing athlete. Phys Sportsmed 2011, 39(4):90-97.

13. Kobayashi H, Koyama Y, Enoka RM, Suzuki S: A unique form of light-load training improves steadiness and performance on some functional tasks in older adults. Scand J Med Sci Sports 2014, 24(1):98-110.

14. McMahon PJ, Debski RE, Thompson WO, Warner JJ, Fu FH, Woo SL: Shoulder muscle forces and tendon excursions during glenohumeral abduction in the scapular plane. J Shoulder Elbow Surg 1995, 4(3):199-208.

15. Escamilla RF, Yamashiro K, Paulos L, Andrews JR: Shoulder muscle activity and function in common shoulder rehabilitation exercises. Sports Med 2009, 39(8):663-685.

16. Lin YL, Karduna A: Four-week exercise program does not change rotator cuff muscle activation and scapular kinematics in healthy subjects. Journal of orthopaedic research : official publication of the Orthopaedic Research Society 2016, 34(12):2079-2088.

17. Kibler WB, Chandler TJ, Shapiro R, Conuel M: Muscle activation in coupled scapulohumeral motions in the high performance tennis serve. Br J Sports Med 2007, 41(11):745-749.

18. Rosso C, Mueller AM, McKenzie B, Entezari V, Cereatti A, Della Croce U, Ramappa AJ, Nazarian A, DeAngelis JP: Bulk effect of the deltoid muscle on the glenohumeral joint. J Exp Orthop 2014, 1(1):14.

19. Gowan ID, Jobe FW, Tibone JE, Perry J, Moynes DR: A comparative electromyographic analysis of the shoulder during pitching. Professional versus amateur pitchers. Am J Sports Med 1987, 15(6):586-590.

20. David G. Simons JGT, Lois S. Simons: David G. Simons: Travell \& Simons' Myofascial Pain and Dysfunction : The Trigger Point Manual: Volume 1: Upper Half of Body: Lippincott Williams \& Wilkins; 1999.

21. Meron A, Saint-Phard D: Track and Field Throwing Sports: Injuries and Prevention. Curr Sports Med Rep 2017, 16(6):391-396.

22. Olsen SJ, 2nd, Fleisig GS, Dun S, Loftice J, Andrews JR: Risk factors for shoulder and elbow injuries in adolescent baseball pitchers. Am J Sports Med 2006, 34(6):905-912.

23. Fortenbaugh D, Fleisig GS, Andrews JR: Baseball pitching biomechanics in relation to injury risk and performance. Sports Health 2009, 1(4):314-320.

24. Bartlett LR, Storey MD, Simons BD: Measurement of upper extremity torque production and its relationship to throwing speed in the competitive athlete. Am J Sports Med 1989, 17(1):89-91.

25. Lehman G, Drinkwater EJ, Behm DG: Correlation of throwing velocity to the results of lower-body field tests in male college baseball players. J Strength Cond Res 2013, 27(4):902-908. 


\section{Figures}

Fig. 1: a. Scapula 2000

b. Scapula 1000

\section{c. Clavicle $\mathbf{2 0 0 0}$}

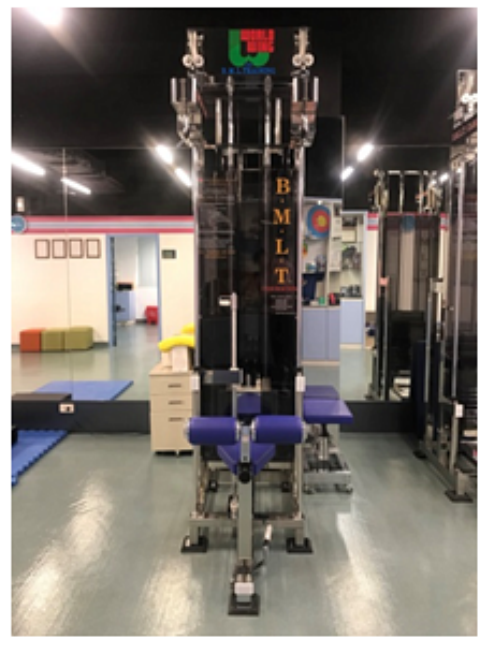

Fig. 1a

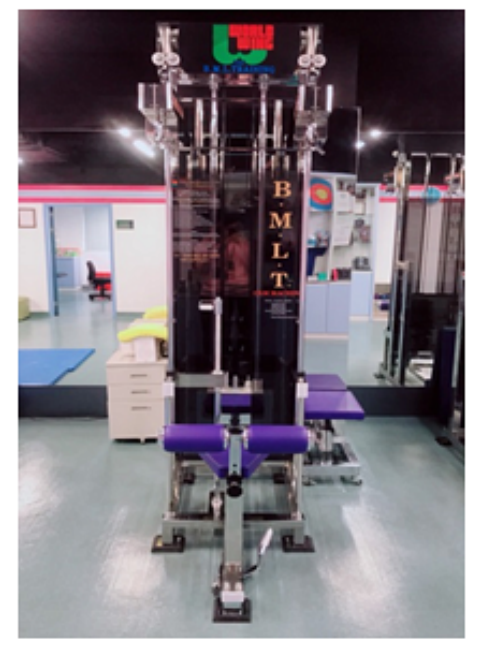

Fig. 1b

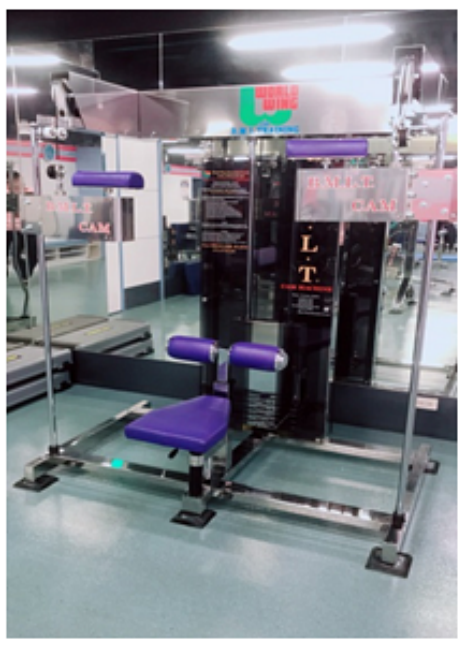

Fig. 1c

\section{Figure 1}

Scapula 2000 (a) Full relaxation (b) full lengthening, flexion (c) Posterior horizontal adduction with contraction 
Fig. 2: Scapula 2000

a. Hand on handle with semi-full stretch, relaxation

b. Hand on handle with full lengthening

c. Shoulder horizontal adduction with muscle contraction

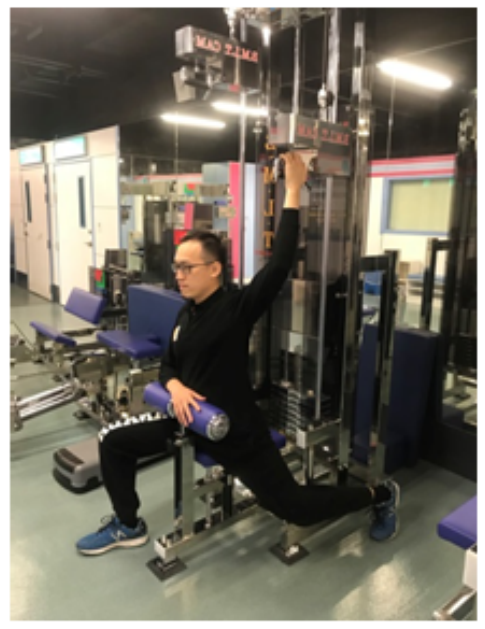

Fig. 2a

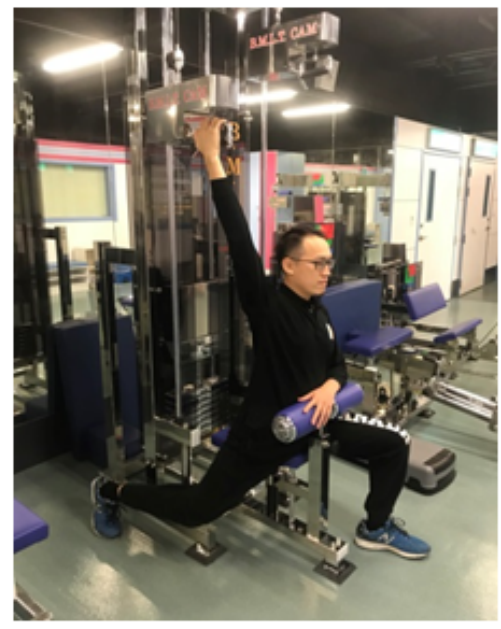

Fig. 2b

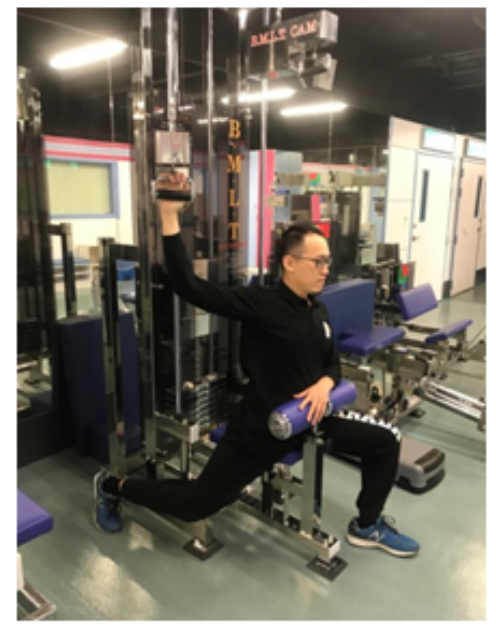

Fig. 2c

Figure 2

Clavicle 2000 (a) shoulder 0 degree abduction; (b) shoulder 90 degree abduction and internal rotation 
Fig. 3: Clavicle 2000

a. Hand on handle with shoulder 0-degree abduction

b. Hand on handle with shoulder 90-degree abduction and internal rotation

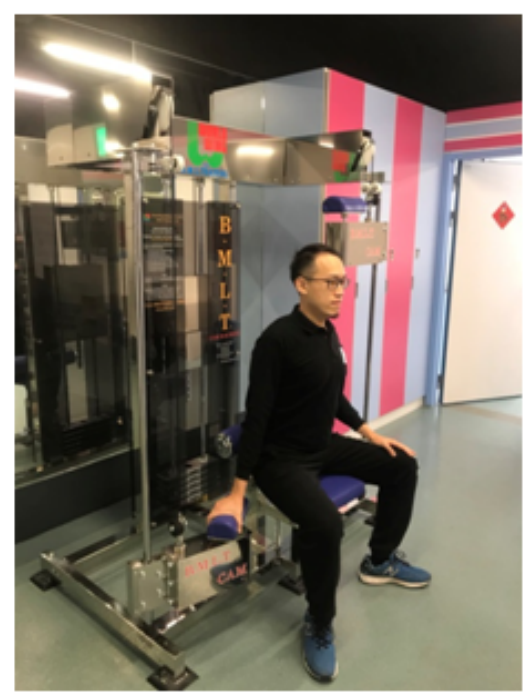

Fig. 3a

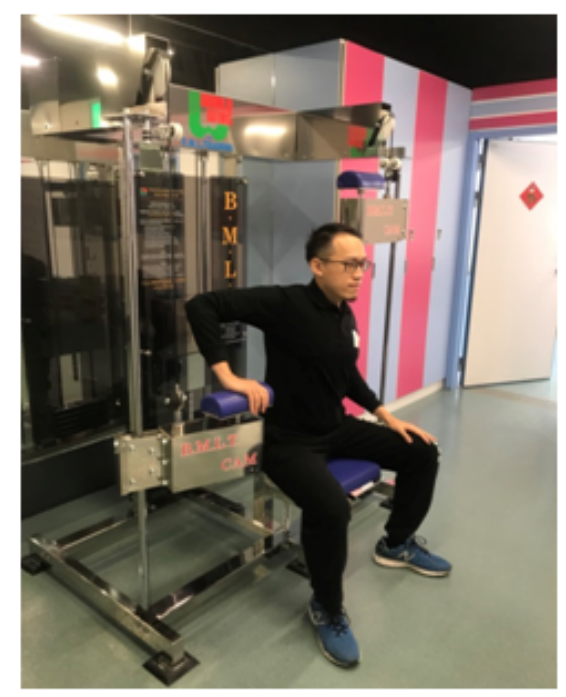

Fig. 3b

Figure 3

(a) Scapula 2000; (b) Scapula 1000; (c) Clavicle 2000 
(a)

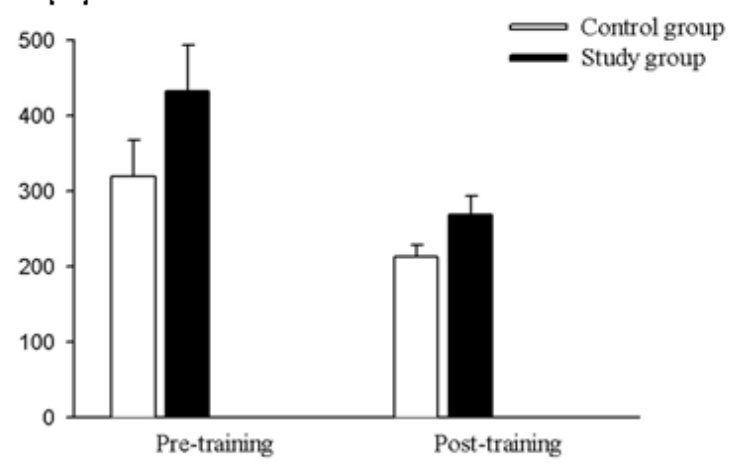

(c) Supraspinatus

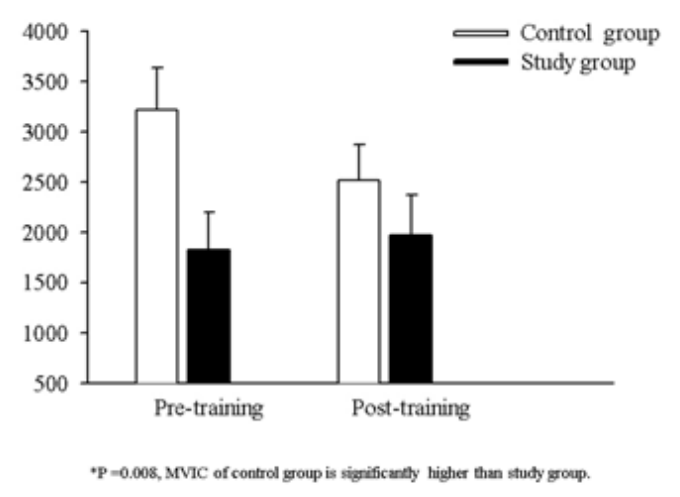

(b)

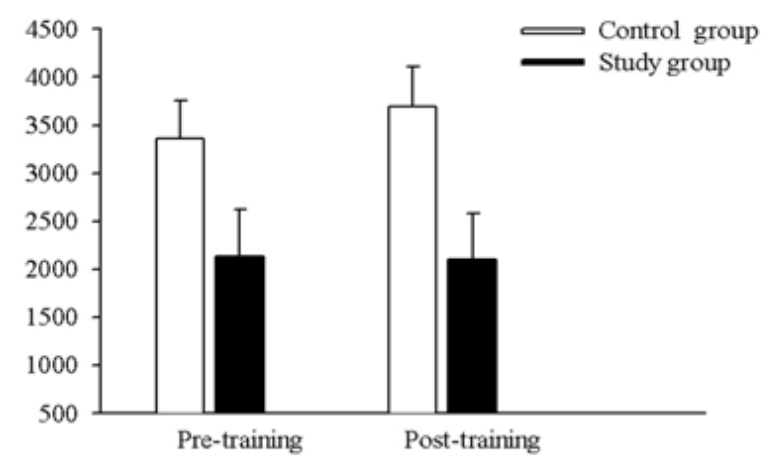

$* \mathrm{P}=0.013 \mathrm{MVIC}$ of control group is significantly higher than study group
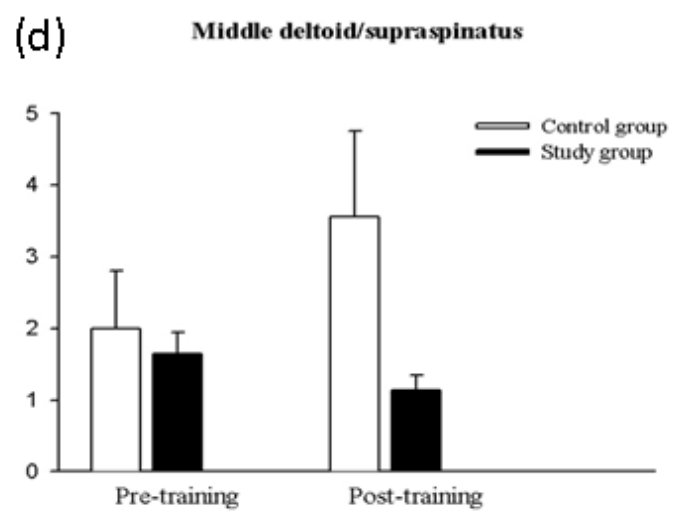

${ }^{*} \mathrm{P}=0.044$, MVIC of control group is significantly highter than study group.

\section{Figure 4}

Maximal voluntary isometric contraction (a) Pectroralic major (b) Middle deltoid (c) Supraspinatus (d) Middle deltoid/supraspinatus 